Portland State University

PDXScholar

Physics Faculty Publications and Presentations

Physics

10-2016

\title{
The Physics of Juggling a Spinning Ping-Pong Ball.
}

Ralf Widenhorn

Portland State University

Follow this and additional works at: https://pdxscholar.library.pdx.edu/phy_fac

Part of the Physics Commons

Let us know how access to this document benefits you.

Citation Details

Widenhorn, R. (2016). The physics of juggling a spinning ping-pong ball. American Journal of Physics, 84(12), 936-942.

This Article is brought to you for free and open access. It has been accepted for inclusion in Physics Faculty Publications and Presentations by an authorized administrator of PDXScholar. Please contact us if we can make this document more accessible: pdxscholar@pdx.edu. 


\title{
The physics of juggling a spinning ping-pong ball
}

\author{
Ralf Widenhorn ${ }^{\text {a) }}$ \\ Department of Physics, Portland State University, Portland, Oregon 97201
}

(Received 14 August 2015; accepted 18 September 2016)

\begin{abstract}
Juggling a spinning ball with a ping-pong paddle represents a challenge both in terms of hand-eye coordination and physics concepts. Here, we analyze the ping-pong ball's motion, and explore how the correct paddle angle relates to the ball's spin and speed, as it moves vertically up and down. For students, this requires engaging with concepts like momentum, angular momentum, free-body diagrams, and friction. The activities described in this article include high-speed video motion tracking of the ping-pong ball and the investigation of the frictional characteristics of the paddle. They can be done in a physics lab or at home, requiring only inexpensive or commonly used equipment, and can be undertaken by high school or college students. (C) 2016 American Association of Physics Teachers. [http://dx.doi.org/10.1119/1.4964104]
\end{abstract}

\section{INTRODUCTION}

Students usually get their first introduction to physics through mechanics. The study of motion provides various opportunities for lab activities. Although students have developed an intuition through everyday experience of how objects move, the challenges for students to correctly understand these concepts have been well documented since the early years of physics education research. ${ }^{1}$ While a standard laboratory experiment aims to teach important concepts and experimental skills, we find few "typical" experiments excite our students. Furthermore, labs frequently suffer from being "cookbook style," with little room for students to actively engage and explore physics phenomena, or to develop true experimental skills that have been identified as important by the American Association of Physics Teachers Recommendations for the Undergraduate Physics Laboratory Curriculum ${ }^{2}$ and the Next Generation Science Standards. ${ }^{3}$ One way to allow students to more freely explore basic physics principles and develop experimental skills is to have them work on a project lab. ${ }^{4} \mathrm{~A}$ good project leaves room for students to explore different aspects of a phenomenon, is challenging, captures a student's interest, and at the same time provides the opportunity to discuss experimental design.

The exploration of physics phenomena in sports can provide an extra stimulus to spark the interest of many students and has been the subject of several textbooks. ${ }^{5,6}$ However, it is difficult to investigate sports activities in a lab environment. Table tennis, often referred to colloquially as pingpong, uses a light ball that can be easily studied in a confined space. A key difference between competitive table tennis and recreational ping-pong is the use of spin. The spin of a ping-pong ball is difficult to observe directly, but its effect on all aspects of the game is profound. The mass of the ball is small and the ball's trajectory and its motion upon bouncing off the table and paddle are non-intuitive for all but the most experienced players (see Fig. 1). While this makes it more difficult to predict a ball's trajectory, it also makes the motion more intriguing to analyze. Concepts like kinematics, projectile motion, free-body diagrams, friction, air resistance, the Magnus force, kinetic energy, rotational kinetic energy, impulse, forces, and angular momentum can all play an important role in such an analysis. Various articles have been written on the bounce of spinning balls in pingpong $^{7-12}$ and other types of spinning balls upon hitting a racquet, paddle, club, or the ground. ${ }^{13-25}$ Following up on these studies, we present a project that many students who enjoy ball sports will find to be a challenge to their hand-eye coordination and their physics skills. For our study we will focus on the effect of spin on the bounce of the ping-pong ball. Due to the relatively small speeds involved we will neglect drag forces ${ }^{26-28}$ and the curving of the ball due to the Magnus force. ${ }^{29-33}$

The goal here is to hit a ping-pong ball upward with some spin, and try to control it when it impacts the paddle to send it straight up again, so that it can easily be caught afterwards. Such an exercise helps a player to get a feel for the speed and tackiness of the paddle. For the study described in this manuscript, we use a standard 40-mm plastic ping-pong ball with a pre-assembled entry level Stiga Inspire paddle with Magic rubber (1.5-mm sponge $)^{34}$ and the competitive grade combo of a Butterfly Tenergy 05 rubber (2.1-mm sponge) ${ }^{35}$ on a Timo Boll Spirit blade. ${ }^{36}$ The lower quality Magic rubber had lost its initial tackiness while the Tenergy rubber was still tacky.

One can send a ball without spin straight up, by placing the paddle flat under the ball, but in the case of a spinning ball the paddle needs to be angled as shown in Fig. 2. With some practice, one can develop a good intuition on how to angle the paddle and juggle the spinning ball multiple times by alternating the angle of the paddle from being titled clockwise to counterclockwise, sending the ball straight up each time. To analyze this motion a couple of research questions might include: "At what angle $\alpha$, does the paddle need to be placed to have the ball go vertically upward for different initial and final speeds and spins of the ball?" and "How does this angle depend on the type of paddle?"

\section{MOTION TRACKING}

We used a point-and-shoot Casio Exilim EX-FH100 camera $^{37}$ that captures video in the high frame rate shooting modes of $240 \mathrm{fps}$ at $448 \times 336$ pixels, and at $1,000 \mathrm{fps}$ at $224 \times 64$ pixels. The compact consumer camera was equipped with an SD card rated for $10 \mathrm{MB} / \mathrm{s}$ and was mounted on a standard tripod. We use Vernier Logger Pro software to extract data from the videos using frame-by-frame tracking of the ball. $^{38}$

For students new to ping-pong, the first step is to practice how to brush the ping-pong ball from underneath with the paddle such that the ball has a large spin and flies up approximately vertically. Next, students will record their attempts 


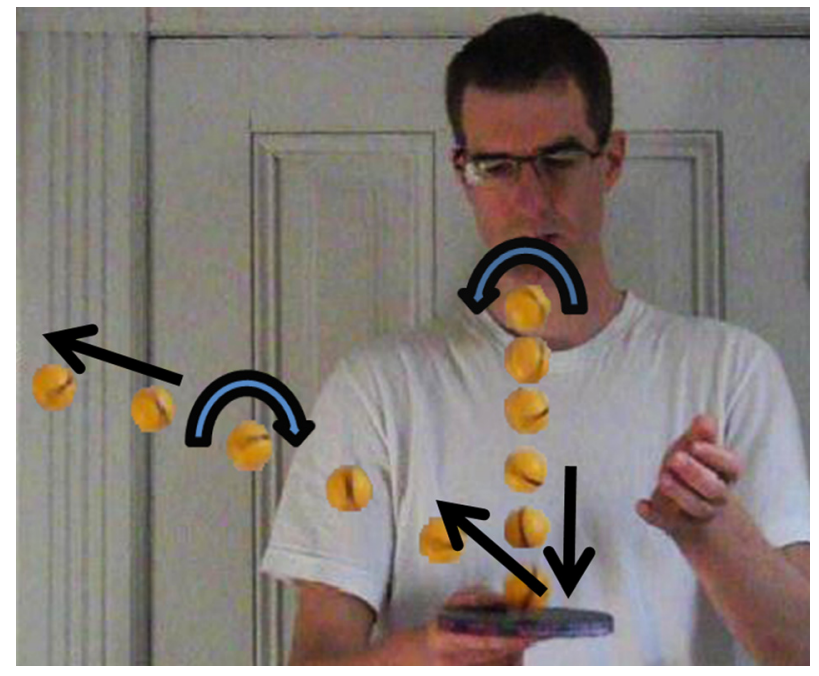

Fig. 1. Overlay image of a spinning ball dropping vertically onto a horizontal paddle. The video was taken at a frame rate of $240 \mathrm{fps}$, and the ball location is shown for every tenth frame, approximately $41.7 \mathrm{~ms}$ apart.

to hold the paddle such that the ball pops straight up, catching the ball after each try. Even though it is a fun challenge, one does not need to juggle the ball multiple times for this project. For the video capture, it is easiest to use a tripod so that one can easily adjust the level and angle of the camera. For the study described in this section, we chose a frame rate of $240 \mathrm{fps}$, which provides sufficient spatial resolution for motion tracking. One finds that the ball velocities for this experiment are on the order of a few meters per second or less and are easily tracked at a lower frame rate. However, the spin can be such that a high frame rate is required to track the rotational speed of the ball. The $240 \mathrm{fps}$ frame rate would have a ball spinning at $60 \mathrm{rev} / \mathrm{s}$ rotate by an easily traceable quarter of revolution from frame to frame. Particularly for students interested in engineering, this would be a good opportunity to explore signal processing, the Nyquist frequency, and aliasing effects. Drawing a black line around the circumference of the ball and adding two dots at the ball's polar opposites allowed the ability to track the ball's spin. The rotational velocity is found by counting the rotations of the line or dots from frame to frame. Video acquisition should be done in a well-lit room, avoiding incandescence and other light sources that exhibit power line flicker.

Figure 1 shows the impact of the spin on the bounce of the ball. The ball rotated counterclockwise (from the perspective of the reader) three times over 36 frames before bouncing off the paddle. Defining a clockwise rotation as positive, the angular velocity of the spinning ball was therefore $\omega_{i}$ $=-240 \mathrm{fps} /(12 \mathrm{f} / \mathrm{rev})=-20 \mathrm{rev} / \mathrm{s}$. Following the bounce, the ball rotated clockwise once over 38 frames, resulting in an angular velocity of $\omega_{f}=6.3 \mathrm{rev} / \mathrm{s}$.

To prevent the ball from bouncing off to the side, one needs to angle the paddle. The counterclockwise rotation of the ball before impact, as shown in Fig. 2(a), requires angling the paddle as depicted in Fig. 2(b). Moving the paddle as indicated with the arrows in Figs. 2(b) and 2(e) will add extra speed and counter-rotation to the ball. Upon impact, the ball rises and then falls, as shown in Figs. 2(c) and 2(d) while now spinning clockwise. A ball with this spin would jump to the right if the paddle is held horizontally, hence the paddle needs to be angled as shown in Fig. 2(d).

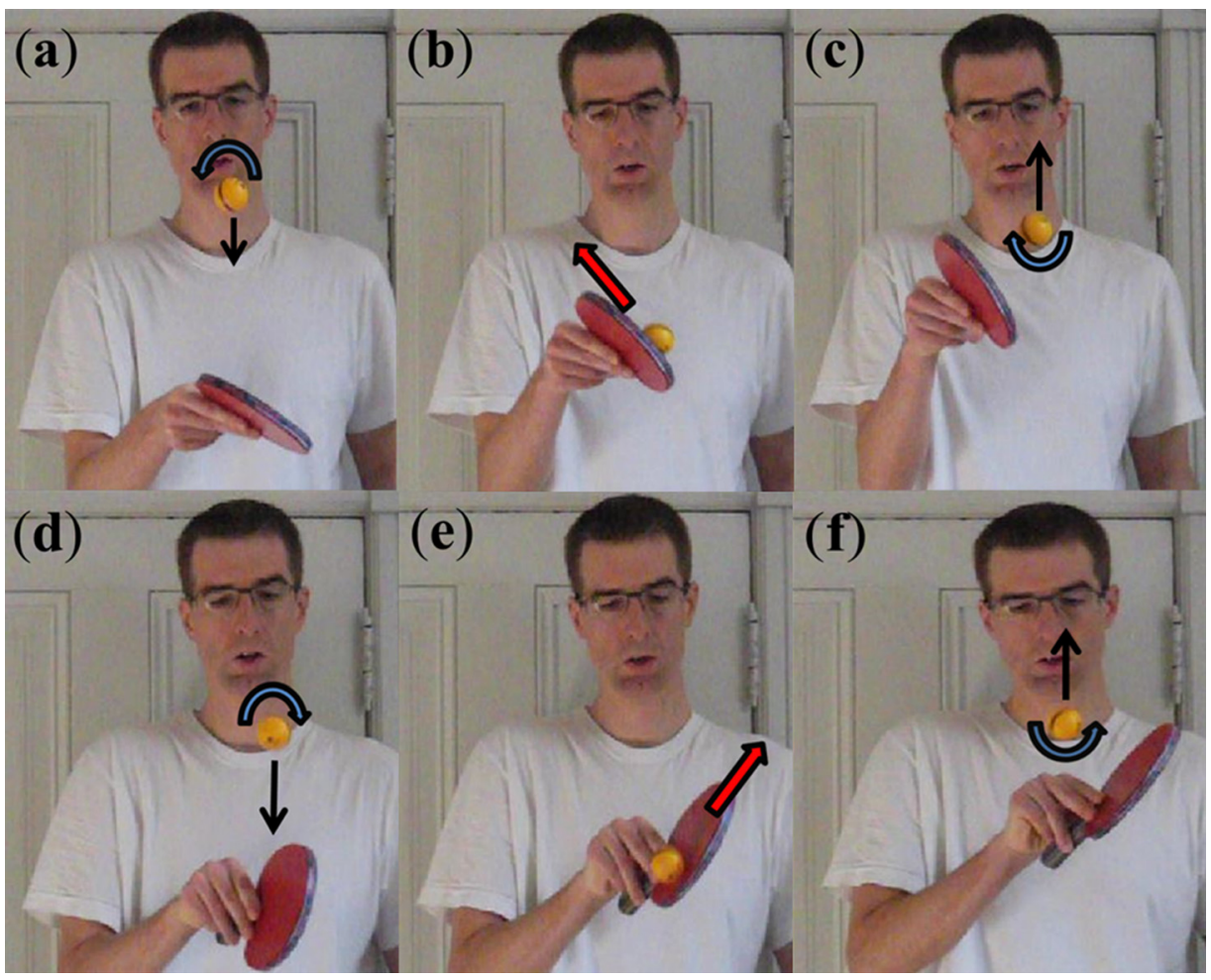

Fig. 2. Sequence of images when juggling a spinning ping-pong ball. The frames were selected to illustrate one complete juggling cycle: (a) ball falling vertically and rotating counterclockwise; (b) angled paddle impacting the ball, the paddle moves in the direction as indicated by the arrow; (c) ball moving vertically upwards after impact while rotating clockwise; (d) ball dropping back vertically toward the paddle while still rotating clockwise; (e) angled paddle impacting the ball, the paddle moves in the direction as indicated by the arrow; (f) ball moving vertically upward after impact while rotating counterclockwise. 
After impact, the ball will move upwards with a counterclockwise spin as shown in Fig. 2(e). A skilled student can repeat the sequence as the ball falls again, as in Fig. 2(a). For the motion analysis, it is sufficient to consider the first three figures as the paddle angle and ball motion of the following part of the sequence are symmetric with rotational directions and the horizontal axis flipped.

To convert the pixel distance in the frame-by-frame tracking of the ball to a physical distance, one needs to calibrate it with an object of known length. The calibration was done by displaying a $50-\mathrm{cm}$ ruler in the plane of motion at one point during the video capture. With this reference distance and the frame rate of the video capture, one can plot horizontal and vertical distances and velocities as a function of time. For this study, the camera was about $3 \mathrm{~m}$ away from the ball, resulting in small angles for the vertical positions analyzed.

Figure 3(a) shows the position during the fall of a ball from close to its peak motion to after it hits the paddle. The ball drops and rises almost vertically with a slight movement to the right throughout its trajectory. The vertical position before impact varies quadratically as a function of time. The best-fit line results in a gravitational acceleration slightly larger than the theoretical value, pointing to the calibration length being slightly off. This can be due the fact that the height of the exact contact point with the ball, and the height
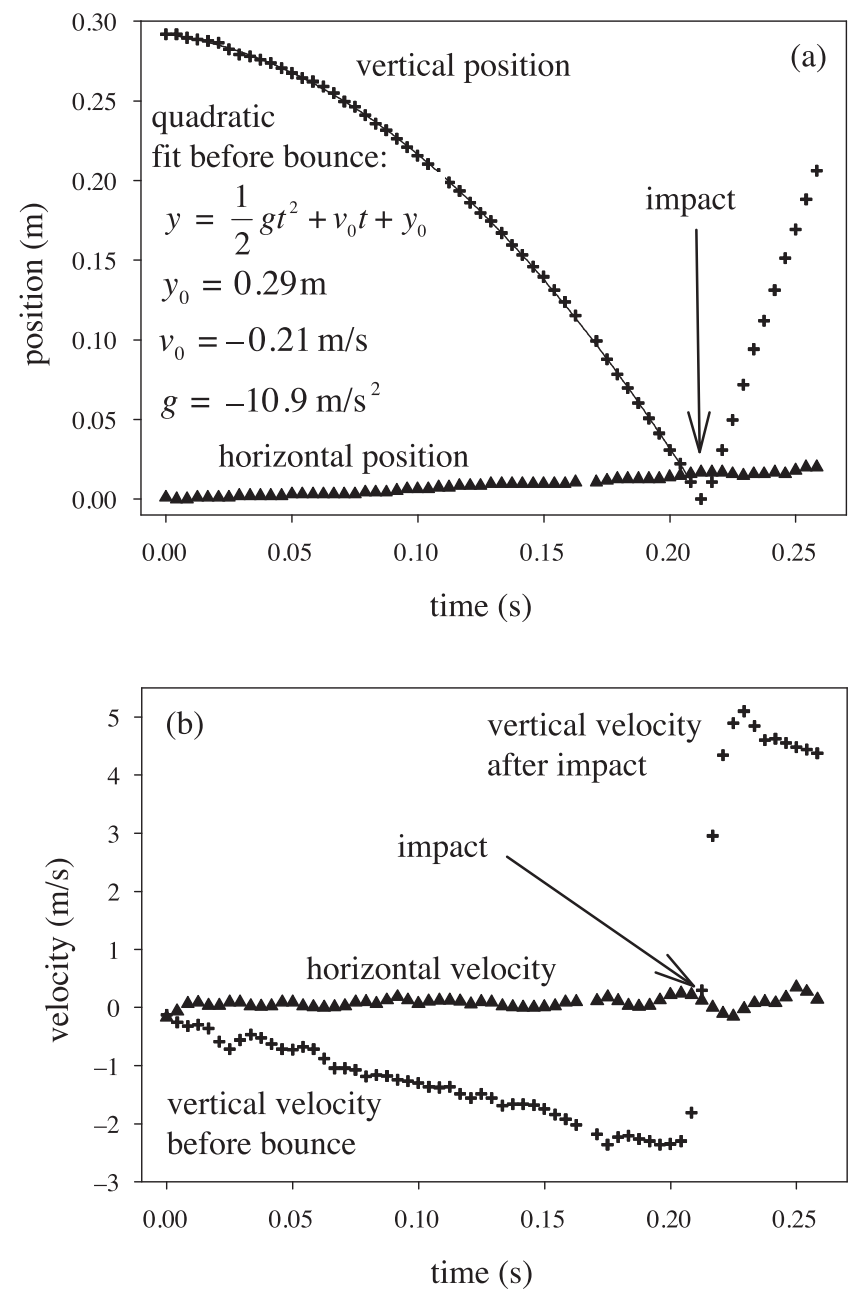

Fig. 3. (a) Frame-by-frame position tracking of the ball in the horizontal and vertical directions. (b) Corresponding horizontal and vertical velocities as a function of time. from which the ball dropped, varied from trial to trial and therefore is not always in line with the position of the ruler during calibration. The exact distance of the plane of motion of the ball from the camera varied from trial to trial as well. Each point on the velocity versus time graph shown in Fig. 3(b) is calculated from a seven frames kernel to calculate the derivative of the position data in Fig. 3(a). This causes the smoothing of the velocity data, which is especially visible for $v_{y}$ around the point of impact.

The data shown in Fig. 3 indicate that the horizontal velocity was small throughout the motion, and the ball successfully bounces almost vertically off the paddle. Under the influence of gravity, the magnitude of the vertical velocity increases linearly with time until the ball hits the paddle and changes direction. The large magnitude of the velocity after impact indicates that the paddle added extra translational kinetic energy to the ball. The velocity $v_{i}$ before and the velocity $v_{f}$ after impact can be obtained from linear fits to the corresponding data points. The data points for the three frames before and after the bounce are not accurate, due to the smoothing of the velocity data and are not included in the fits. The small changes in the horizontal velocity further indicate that the speed of the ball is small enough so that the Magnus force did not have a significant impact on the trajectory.

\section{MOTION ANALYSIS}

To analyze the motion of the ball, one needs to consider both its spin and linear velocity. Figure 4(a) shows all forces acting on the ball on impact. The weight of the ball is included for pedagogical reasons though for most cases it will be small compared to the other forces during impact.

Choosing the center of the ping-pong ball as the rotational axis, we can calculate the change in angular momentum by multiplying the torque $\tau$ by the time over which it acts $\Delta t$, giving

$$
\Delta L=\tau \Delta t=F_{f} r \Delta t=F r \Delta t \sin \alpha,
$$

where $r$ is the radius of the ball (see Fig. 4 for the definitions of $F_{f}, F$, and $\alpha$ ). Meanwhile, the change in linear momentum in the vertical direction is

$$
\Delta p=F \Delta t-F_{g} \Delta t
$$

which can be expressed as (a)

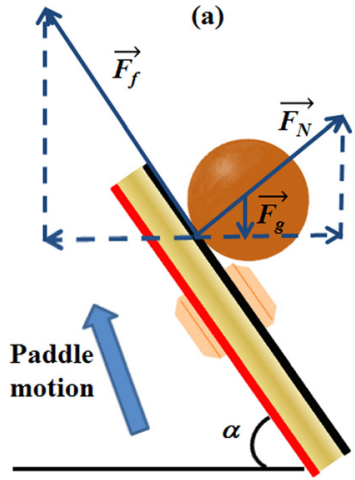

(b)

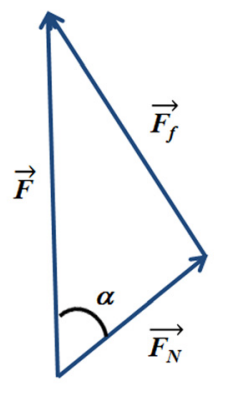

(c)

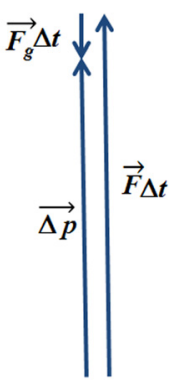

Fig. 4. (a) Forces acting on the ball as it bounces off the paddle upon impact. (b) The vector sum of the frictional force $F_{f}$ and the normal force $F_{N}$ result in a vertical net force on the ball. (c) Impulse and change in momentum of the ball. 


$$
F=\frac{\Delta p}{\Delta t}+F_{g}
$$

Inserting Eq. (3) into Eq. (1) then gives

$$
\Delta L=\left(\frac{\Delta p}{\Delta t}+F_{g}\right) r \Delta t \sin \alpha,
$$

and solving for $\alpha$ results in

$$
\sin \alpha=\frac{\Delta L}{r \Delta t\left(\Delta p / \Delta t+F_{g}\right)} .
$$

Since the weight of the ping-pong ball is small and in most cases

$$
\Delta p / \Delta t \gg F_{g},
$$

this result becomes

$$
\sin \alpha=\frac{\Delta L}{r \Delta p} .
$$

We note that in vector notation this equation can be expressed simply as $\Delta \vec{L}=\vec{r} \times \Delta \vec{p}$. To find the angle $\alpha$ in terms of the measurable quantities $\Delta \omega$ and $\Delta v$, we need to replace $\Delta p$ and $\Delta L$ in Eq. (7). Though one could include the thickness of the ping-ping ball shell, ${ }^{39,40}$ we are assuming the ping-pong ball has the moment of inertia of a hollow sphere so the change in angular momentum can be calculated using

$$
I=\frac{2}{3} m r^{2}
$$

giving

$$
\Delta L=I\left(\omega_{f}-\omega_{i}\right)=\frac{2}{3} m r^{2} \Delta \omega .
$$

Using

$$
\Delta p=m\left(v_{f}-v_{i}\right)=m \Delta v
$$

and inserting Eqs. (9) and (10) into Eq. (7) results in

$$
\sin \alpha=\frac{2 r \Delta \omega}{3 \Delta v} .
$$

The minimum required coefficient of friction of the rubber sheet at the angle $\alpha$ can then be calculated (see Fig. 4) from

$$
\mu_{\min }=\frac{F_{f}}{F_{N}}=\tan \alpha .
$$

Table I shows a set of data taken with both paddles. Trials 1 and 2 attempted to move the paddle very little on impact and still have the ball bounce upward. One can observe that for both paddles, the ball bounces off with a slightly smaller speed. The elastic Tenergy rubber sheet reverses the spin almost completely. Other trials, with little movement of the paddle, showed $\omega_{f}$ is generally slightly smaller than $\omega_{i}$, but overall confirmed that most of the spin is inverted and therefore has a large tangential coefficient of restitution (ratio of the outgoing and incoming velocity tangential to the ball surface)
Table I. Paddle angle for different vertical and angular velocities. The quantities $\omega_{i}, \omega_{f}, v_{i}$, and $v_{f}$ are determined from motion tracking, $\alpha_{\text {measured }}$ is found from position measurements on the frame of impact, $\alpha$ is calculated using Eq. (11), and $\mu_{\min }$ is found from Eq. (12).

\begin{tabular}{lccccccccc}
\hline \hline Trial & Rubber & $\begin{array}{c}\omega_{i} \\
(\mathrm{rev} / \mathrm{s})\end{array}$ & $\begin{array}{c}\omega_{f} \\
(\mathrm{rev} / \mathrm{s})\end{array}$ & $\begin{array}{c}v_{i} \\
(\mathrm{~m} / \mathrm{s})\end{array}$ & $\begin{array}{c}v_{f} \\
(\mathrm{~m} / \mathrm{s})\end{array}$ & $\begin{array}{c}\alpha \\
(\mathrm{deg})\end{array}$ & $\begin{array}{c}\alpha_{\text {measured }} \\
(\mathrm{deg})\end{array}$ & $\begin{array}{c}\% \\
\text { diff. }\end{array}$ & $\begin{array}{c}\mu_{\min } \\
=\tan \alpha\end{array}$ \\
\hline 1 & Tenergy & -17 & 17 & -3.7 & 3.2 & 24 & 20 & 16 & 0.44 \\
2 & Magic & -18 & 2 & -2.4 & 2.2 & 20 & 19 & 8 & 0.37 \\
3 & Tenergy & -12 & 37 & -3.2 & 4.1 & 34 & 34 & 0 & 0.67 \\
4 & Tenergy & -28 & 41 & -2.4 & 5 & 51 & 52 & -2 & 1.22 \\
5 & Tenergy & -18 & 25 & -2.1 & 2.2 & 54 & 45 & 17 & 1.37 \\
6 & Magic & -12 & 11 & -2.1 & 2.6 & 25 & $24^{\circ}$ & 3 & 0.46 \\
\hline \hline
\end{tabular}

for this rubber sheet. For the Magic rubber, there is almost no spin after the bounce. It was generally found that little paddle movement resulted in a small inverted spin corresponding to a tangential coefficient of restitution of close to zero for this rubber sheet. The angle $\alpha$ is calculated using Eq. (11) and compared with $\alpha_{\text {measured }}$, which is determined by measuring the physical placement of the paddle in the frame of impact using a virtual ruler in Logger Pro.

If we want to send a ball with $\omega_{i}$ and $v_{i}$ vertically upwards to the same level, with little movement of the paddle, we can determine the angle at which to place the paddle: $\Delta v$ needs to be equal to $2 v_{i}$ to reach the same level. For the Tenergy rubber, we can approximate $\Delta \omega=2 \omega_{i}$ and Eq. (11) results in

$$
\sin \alpha=\frac{2 r \omega_{i}}{3 v_{i}} .
$$

Meanwhile, in a first approximation, the ball loses most of its rotation upon impact for the Magic rubber sheet. Assuming $\omega_{f}=0$ Eq. (11) leads to

$$
\sin \alpha=\frac{r \omega_{i}}{3 v_{i}}
$$

Sending the ball back to the same level with little movement of the paddle therefore requires angling the tacky and elastic Tenergy rubber paddle at a larger angle than the paddle with the Magic rubber. For the Tenergy paddle, the rotation is inverted and one could juggle a ball as often as one likes up and down by alternating the paddle angle from $+\alpha$ to $-\alpha$. The Magic rubber causes the ball to lose most of its spin, and one could place the paddle almost horizontally on the next stroke.

For both paddles, we can vary the spin and velocity of the ball by striking it with a greater paddle speed. Depending on the direction of the paddle motion, one imparts more spin or translational velocity on the ball. As long as Eq. (11) is satisfied, the ball will travel straight up. By increasing the spin, one increases the change in angular momentum and hence the paddle angle. Trials 3 and 4 both increase the spin and translational velocity. A large change in the angular velocity, as in trial 4, will result in a more angled paddle. Trial 5 is an example of a slow ball being sent back to roughly the same level with a slight increase in rotational kinetic energy and a strongly angled paddle. Trial 6 shows that one can invert the spin with the Magic rubber paddle, however, this requires moving the paddle quickly in the direction of the angle $\alpha$. The result for the Magic rubber demonstrates that one can continuously juggle the ball if one adds a significant swing 
of the paddle. Thus, even though the paddle angle for the Magic and Tenergy rubber would be identical for the same $\Delta \omega$ and $\Delta v$, the motion of the paddle would be quite different. The necessary fast swing of the Magic paddle along the direction of $\alpha$ makes juggling the ball with the Magic paddle more difficult and requires more effort by the player than with the Tenergy paddle where a slow motion at the correct angle is sufficient.

Note that in Table I, the experimental difference between the measured and calculated angles is largest for trials 1 and 5. In both of these cases $\alpha$ is larger than $\alpha_{\text {measured }}$ A larger $\alpha$ corresponds to a larger change in the horizontal velocity and for these two trials the trajectory was the least vertical, with a horizontal velocity change of $0.3-0.5 \mathrm{~m} / \mathrm{s}$. The impact of the earlier mentioned slight calibration error appears to be minor, but being successful in getting the ball going straight up and down impacts the agreement of theory and experimental data more strongly. We think the data presented here are what one can reasonably expect from students, though some dedicated students with great hand-eye coordination may be able to get a lower experimental difference.

Equation (11) restricts all solutions to $\Delta \omega<3 \Delta v / 2 r$. However, while all possible solutions must satisfy Eq. (11), for large angles the normal force decreases and the required frictional force may exceed the maximum friction that can be supplied by the paddle rubber. Hence, Eq. (11) is a necessary but not sufficient condition. We will try in Secs. IV and V to estimate the required coefficient of friction of the rubber sheet necessary to exert a large enough frictional force.

\section{TIME OF CONTACT}

To observe the impact of the ball, we set the frame rate to $1,000 \mathrm{fps}$. The camera was placed right next to the paddle and captured the impact of a ball dropped with little spin from a height of $0.5-1 \mathrm{~m}$ with the paddle placed horizontally and angled at $45^{\circ}$. We found that the impact for both paddle angles showed similar results at this temporal resolution. The high frame rate, and therefore short integration times, required good lighting conditions.

The images in Fig. 5 were taken using natural sunlight and the Butterfly paddle placed horizontally. From the video images, using the size of the ball as a reference, one can estimate the distance of the ball from the paddle. From these distances and the inspection of the images close to impact, one can get a rough estimate of the contact time. Of the eight drops we looked at, two showed contact in only one frame (like the left sequence in Fig. 5), while two trials showed contact in two frames (like the right sequence in Fig. 5). The other four trials had one frame with clear contact and another frame so close that the ball may or may not have been in contact with the paddle. The fact that there were trials with only a single image showing full contact places an upper limit for the contact time at $2 \mathrm{~ms}$. The trials showing two sequential images with contact place a lower limit for the contact time at $1 \mathrm{~ms}$. For contact times of 1-2 ms and typical changes in speed of $4-8 \mathrm{~m} / \mathrm{s}, \Delta p / \Delta t$ for the $2.7-\mathrm{g}$ ping-pong ball is in the range of approximately $5-20 \mathrm{~N}$, at least two orders of magnitude larger than $F_{g}$, thus satisfying the condition that $\Delta p / \Delta t \gg F_{g}$. The slight downward impulse due to gravity shown in Fig. 4(c) is therefore indeed negligible. We can calculate the normal force on the ping-pong ball as $F_{N}=(\Delta p / \Delta t) \cos \alpha$, and knowing the order of magnitude of
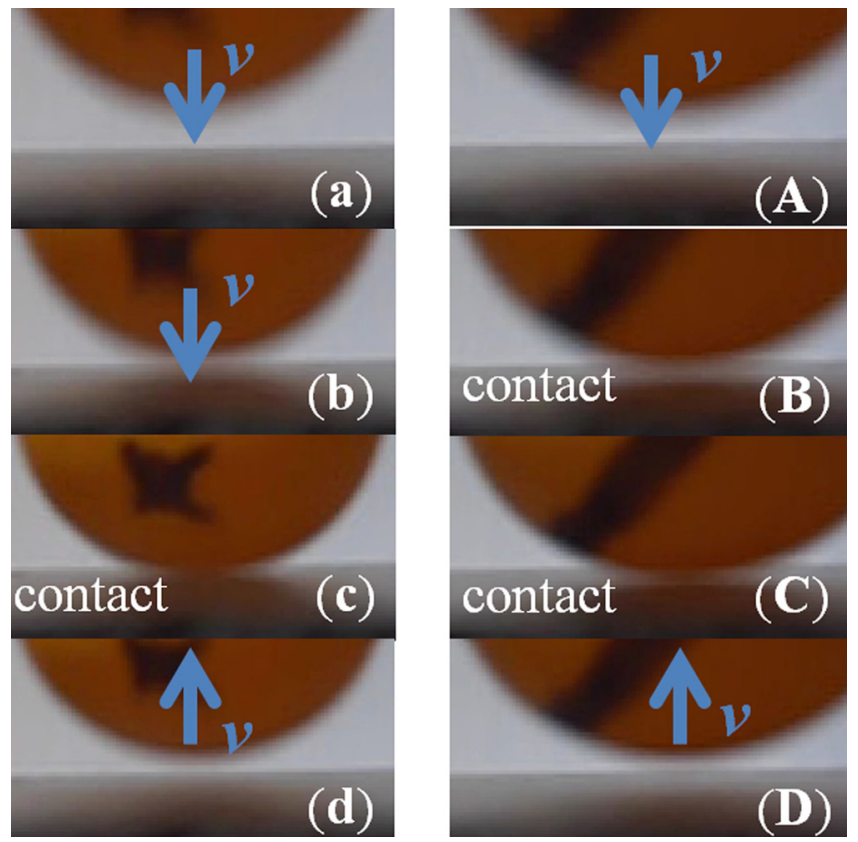

Fig. 5. High speed capture (1,000 fps) of two drops on the Tenergy paddle. The ball had little spin and both the paddle and camera were angled horizontally. The images are sequential starting with frames (a/A) and ending with frames $(d / D)$ with $1 \mathrm{~ms}$ between frames. The ball is in contact with the paddle in frame (c) on the left and in frames (B) and (C) on the right.

the forces during contact with the paddle, we can investigate the frictional forces supplied by the paddle.

\section{FRICTION}

The normal force exerted on the ball by the paddle varies quickly during impact. For this study, we did not obtain time resolved force versus time data and we need to make some simplifying assumptions. We ignore any dependence of the contact time on the paddle angle, speed, and type, as well as the speed and spin of the ball. With the rough estimate of contact time, we can estimate normal and frictional forces if we know the coefficient of friction of the rubber sheet.

For this, we conducted a classical friction experiment by sliding the ping-pong ball, with different weights added, across the surface of interest (the rubber sheet). Figure 6 shows the experimental setup. The ping-pong ball was placed in a measuring cup and fixed with masking tape so that it could not rotate. The total mass of the tape, ball, and measuring cup was $61 \mathrm{~g}$. A 50-g weight hanger is attached for all but the measurement with the lowest normal force. Additional masses, up to a total of $1,511 \mathrm{~g}$, are added in 100-g increments. The weight hanger is stabilized with minimal vertical force with one hand while the other hand applies a horizontal force that is measured with a force sensor. The force is increased until the ball starts to slide for a short distance of $1-3 \mathrm{~cm}$, and this is repeated at least six times. The average and standard deviation of the peak force of six measurements is calculated and plotted as a function of the normal force in Fig. 7(a).

For many surfaces, the maximum frictional force increases linearly with the normal force, with the coefficient of friction $\mu$ as the constant of proportionality. However, the elastic rubber sheet has a coefficient of friction that depends on the normal force. ${ }^{41}$ The normal-force-dependent frictional 


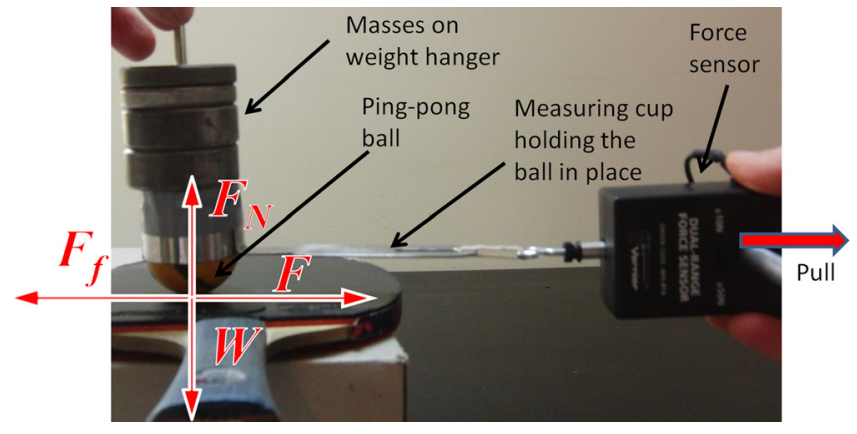

Fig. 6. Setup and free-body diagram for the measurement of the maximum frictional force for different normal forces.

coefficient is calculated as the ratio $F_{f} / F_{N}$ and is plotted in Fig. 7(b). The data for both rubber sheets can be fitted empirically with a power law of the form

$$
\mu\left(F_{N}\right)=2.1 F_{N}^{-0.19} \quad(\text { Tenergy })
$$

and

$$
\mu\left(F_{N}\right)=0.6 F_{N}^{-0.15} \quad(\text { Magic }),
$$
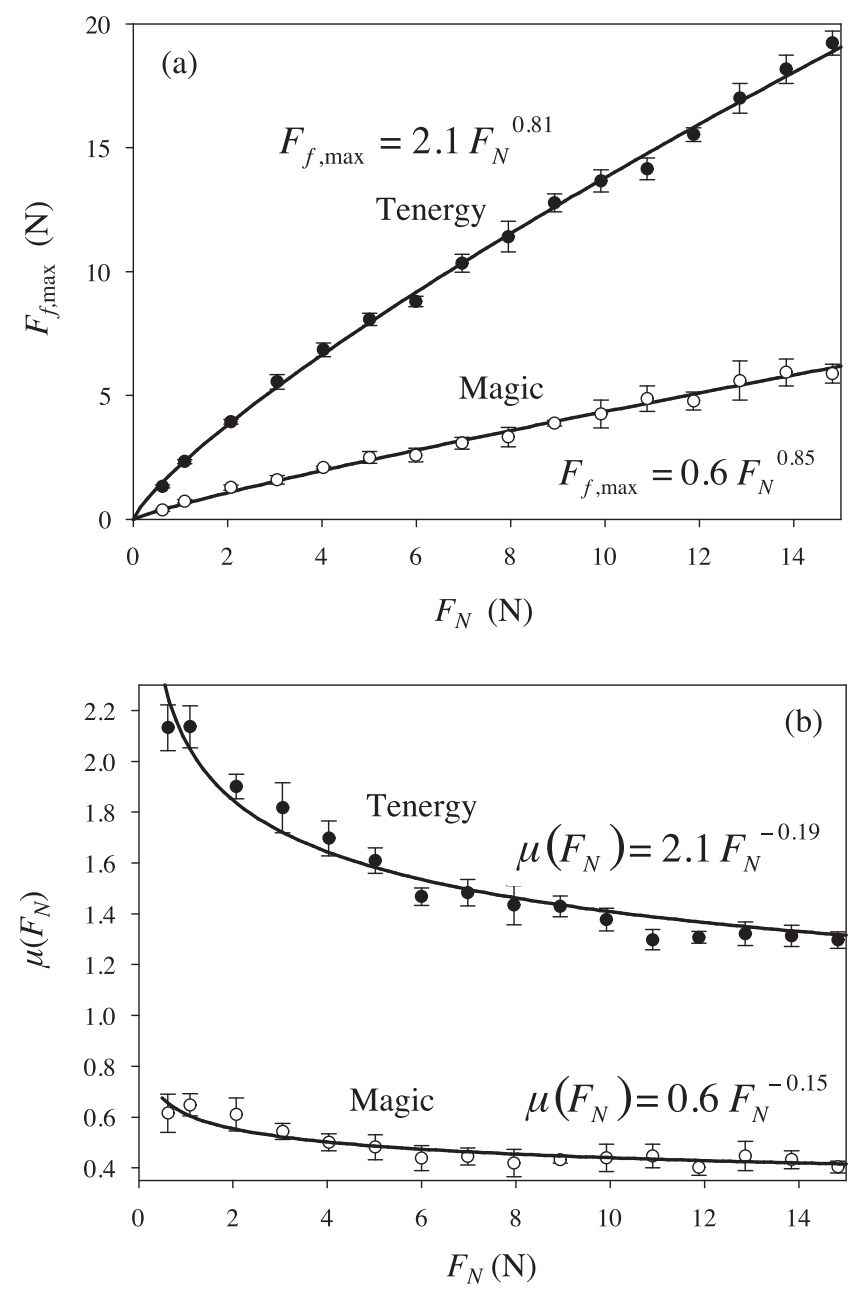

Fig. 7. (a) Maximum frictional force of the Tenergy and Magic rubber sheets for different normal forces. (b) Ratio of the maximum frictional force $F_{f \text {, max }}$ to the normal force $F_{N}$ versus normal force; this ratio represents the effective coefficient of friction for a particular normal force. where $F_{N}$ is measured in Newtons. For example, for a small normal force of $1 \mathrm{~N}$ the frictional coefficient for the Tenergy is 2.1 , answering the classic physics classroom question if $\mu$ can be larger than one. The Tenergy coefficient of friction decreases for larger normal forces to about 1.3 at $F_{N}=15 \mathrm{~N}$. For the same normal forces, the Magic rubber sheet has a coefficient of friction of about 0.6 and 0.4 , respectively.

The last column in Table I shows that for trials 1 and 3 the actual coefficient of friction of the Tenergy rubber vastly exceeds the $\mu_{\min }$ values of 0.44 and 0.67 . Because of the larger angle of the Tenergy paddle for trials 4 and 5, the required $\mu_{\text {min }}$ values of 1.22 and 1.37 are much closer to the actual frictional coefficient. Meanwhile, for both trials with the Magic rubber, the coefficients of friction are such that $\mu_{\min }$ is on the order of the actual $\mu$. The small maximum frictional force is barely sufficient even for the small angles in these trials, which is reflected in practice by the difficulty of juggling the ball with large paddle angles for the Magic rubber. On the other hand, the larger frictional coefficient of the Tenergy paddle gives it the feel of more control even for larger angles. The largest paddle angles can be obtained for a combination of small $\Delta \omega, \Delta v$ pairs, taking advantage of the higher coefficient of friction for small normal forces. To accomplish this, one would need to brush the ball close to the top of its trajectory at a large angle; this would give the ball a small velocity change, effectively juggling the ball almost in place.

\section{CONCLUSION}

We demonstrated that one can investigate the juggling of a spinning ping-pong ball with different paddles using basic concepts from high school or college level introductory physics and inexpensive and commonly available lab equipment. A study like this would be an ideal project for students who enjoy ball sports. Further studies could include the investigation of contact time, coefficients of restitution, and force, with higher temporal resolution for different speeds, angles, spins, paddles, and balls. Moreover, motion analysis could be used to explore how the speed and direction of the paddle motion during impact with the ball influences the change in linear and angular velocities. The activities described here are both welldefined and rich in interesting open-ended research questions. The measurements require both experimental skill and application of physics that spans most concepts of mechanics in a way that we hope will be engaging to many students.

\section{ACKNOWLEDGMENTS}

The author wants to acknowledge Grace Van Ness, Michael Fitzgibbons, Pure Pong in the Pearl, and the anonymous reviewers for their support and helpful feedback.

a)Electronic mail: ralfw@pdx.edu

${ }^{1}$ D. Hestenes, M. Wells, and G. Swackhamer, "Force concept inventory," Phys. Teach. 30, 141-158 (1992).

${ }^{2}$ AAPT Recommendations for the Undergraduate Physics Laboratory Curriculum <https://www.aapt.org/Resources/upload/LabGuidlines Document_EBendorsed_nov10.pdf> (accessed November 19, 2015).

${ }^{3}$ Next Generation Science Standards <http://www.nextgenscience.org/> (accessed June 18, 2016).

${ }^{4}$ P. Gluck and J. King, Physics Project Lab (Oxford U.P., UK, 2015); available at https://www.amazon.com/Physics-Project-Lab-Paul-Gluck/dp/ 0198704585 and http://global.oup.com/about/?cc=us.

${ }^{5} \mathrm{~V}$. McInnes Spathopoulos, An Introduction to the Physics of Sports (Independent Publishing Platform, 2013) available at https:// 
www.amazon.com/Introduction-Physics-Vassilios-McInnes-Spathopoulos/ dp/1483930076 and https://www.createspace.com/.

${ }^{6} \mathrm{M}$. A. Lisa, The Physics of Sports (McGraw-Hill Higher Education, Columbus, OH, 2015); available at https://www.amazon.com/PhysicsSports-Michael-Lisa/dp/0073513970 and http://www.mheducation.com/.

${ }^{7}$ A. Nakashima, Y. Ogawa, Y. Kobayashi, and Y. Hayakawa, "Modeling of rebound phenomenon of a rigid ball with friction and elastic effects," Proceedings of IEEE American. Control Conference (2010), pp. 1410-1415.

${ }^{8}$ S. Araki, S. Sato, and H. Yamazaki, "Collisional properties of ball-racket interactions in terms of normal and tangential coefficients of restitution," Int. J. Table Tennis Sci. 3, 17-49 (1996).

${ }^{9}$ L. Pauchard and S. Rica, "Contact and compression of elastic spherical shells: the physics of a ping-pong ball," Philos. Mag. B 78(2), 225-233 (1998).

${ }^{10} \mathrm{~K}$. Tiefenbacher and A. Durey, "The impact of the table tennis ball on the racket (backside coverings), ” Int. J. Table Tennis Sci. 2, 1-14 (1994).

${ }^{11}$ C. M. Graney, "Taking a swat at physics with a ping-pong paddle," Phys. Teach. 32, 94-98 (1994).

${ }^{12}$ K. Kamijima, Y. Ushiyama, T. Yasaka, and M. Ooba, "Effect of different playing surfaces of the table on ball bounces in table tennis," The 13th ITTF Sports Science Congress May 11-12, Paris, France (2013), pp. 53-56.

${ }^{13}$ R. B. Clark, "That's the way the bouncing ball spins," Phys. Teach. 44, 550-551 (2006).

${ }^{14}$ P. Knipp, "Bouncing balls that spin,” Phys. Teach. 46, 95-96 (2008).

${ }^{15}$ A. Domnech, "A classical experiment revisited: The bounce of balls and superballs in three dimensions," Am. J. Phys. 73, 28-36 (2005).

${ }^{16}$ R. Cross, "Enhancing the bounce of a ball," Phys. Teach. 48, 450-452 (2010).

${ }^{17}$ R. Cross, “The bounce of a ball,” Am. J. Phys. 67, 222-227 (1999).

${ }^{18}$ R. Cross, "Grip-slip behavior of a bouncing ball," Am. J. Phys. 70, 1093-1102 (2002).

${ }^{19}$ R. Cross, "Measurements of the horizontal coefficient of restitution for a superball and a tennis ball," Am. J. Phys. 70, 482-489 (2002).

${ }^{20}$ R. Cross, "Impact of a ball with a bat or racket," Am. J. Phys. 67, 692-702 (1999).

${ }^{21} \mathrm{R}$. Cross, "The coefficient of restitution for collisions of happy balls, unhappy balls, and tennis balls," Am. J. Phys. 68, 1025-1031 (2000).

${ }^{22} \mathrm{R}$. Cross, "Bounce of a spinning ball near normal incidence," Am. J. Phys. 73, 914-920 (2005).
${ }^{23}$ W. A. Turner and G. W. Ellis, "The energetics of a bouncing ball," Phys. Teach. 37, 496-498 (1999).

${ }^{24} \mathrm{H}$. Brody, "That's how the ball bounces," Phys. Teach. 22, 494-497 (1984).

${ }^{25} \mathrm{P}$. A. Maurone and F. J. Wunderlich, "Bouncing ball experiment," Am. J. Phys. 46, 413-415 (1978).

${ }^{26} \mathrm{M}$. Nagurka, "Aerodynamic effects in a dropped ping-pong ball experiment," Int. J. Eng. Educ. 19(4), 623-630 (2003).

${ }^{27}$ M. E. Brandan, M. Gutiérrez, R. Labbé, and A. Menchaca-Rocha, "Measurement of the terminal velocity in air of a ping pong ball using a time to amplitude converter in the millisecond range," Am. J. Phys. 52, 890-893 (1984).

${ }^{28}$ R. M. French, "Part 4: Dropping a ping-pong ball," Exp. Tech. 30(2), 59-60 (2006).

${ }^{29}$ C. Clanet, "Sports ballistics," Annu. Rev. Fluid Mech. 47, 455-478 (2015).

${ }^{30}$ F. Yamamoto, Y. Tsuji, G. Chen, M. Ogawa, and M. Nakagawa, "Basic theory and experiment for the simulation of ball trajectory," Int. J. Table Tennis Sci. 3, 1-15 (1996).

${ }^{31}$ F. Yamamoto, J. Kasai, H. Hirakawa, S. Someya, and K. Okamoto, "Highspeed video image analysis of air flow around a table tennis ball," Int. J. Table Tennis Sci. 6, 149-150 (2010).

${ }^{32} \mathrm{~K}$. Ou, P. Castonguay, and A. Jameson, "Computational sports aerodynamics of a moving sphere: Simulating a ping pong ball in free flight," in 29th AIAA Applied Aerodynamics Conference (2011), pp. 1-16.

${ }^{33}$ Y. Huang, D. Xu, M. Tan, and H. Su, "Trajectory prediction of spinning ball for ping-pong player robot," IEEE/RSJ International Conference on Intelligent Robots and Systems (2011), pp. 3434-3439.

${ }^{34}$ More information on the Stiga Inspire can be found at $<$ http://stigatabletennis.com/en/products/inspire/> (accessed June 18, 2016).

${ }^{35}$ More information on Tenergy rubber can be found at $<$ http://shop.butterflyonline.com/tenergy-05> (accessed June 18, 2016).

${ }^{36}$ More information on the Timo Boll Spirit blade can be found at $<\mathrm{http} / / /$ shop.butterflyonline.com/timo-boll-spirit> (accessed June 18, 2016).

${ }^{37}$ More information on the Casio EX-FH100 can be found at $<$ http:// www.casio-intl.com/asia-mea/en/dc/ex_fh100/> (accessed June 18, 2016).

${ }^{38}$ More information about Logger Pro can be found at $<$ http://www.vernier.com/products/software/lp/> (accessed November 19, 2015).

${ }^{39}$ X. Cao, "Moment of inertia of a ping-pong ball," Phys. Teach. 50, 292 (2012).

${ }^{40}$ J. Mallinckrodt, "Errant ping-pong ball," Phys. Teach. 50, 389 (2012).

${ }^{41}$ A. Schallamach, "The load dependence of rubber friction," Proc. Phys. Soc. B 65, 657-661 (1952).

\section{ALL BACK ISSUES ARE AVAILABLE ONLINE}

The contents of the American Journal of Physics are available online. AJP subscribers can search and view full text of AJP issues from the first issue published in 1933 to the present. Browsing abstracts and tables of contents of online issues and the searching of titles, abstracts, etc. is unrestricted. For access to the online version of AJP, please visit http://aapt.org/ajp.

Institutional and library ("nonmember") subscibers have access via IP addresses to the full text of articles that are online; to activate access, these subscribers should contact AIP, Circulation \& Fulfillment Division, 800-344-6902; outside North American 516-576-2270 or subs@aip.org.

APPT (individual) members also have access to the American Journal of Physics Online. Not a member yet? Join today http://www.aapt.org/membership/joining.cfm. Sign up for your free Table of Contents Alerts at http://www.ajp.aapt.org/features/toc_email_alerts. 